\title{
SIMULATION OF FLUID-STRUCTURE INTERACTION AND IMPACT FORCE ON A REED VALVE
}

\author{
P. Castrillo*1 E. Schillaci $^{2}$ and J. Rigola ${ }^{1}$ \\ ${ }^{1}$ Heat and Mass Transfer Technological Center (CTTC), Universitat Politècnica de Catalunya \\ BARCELONA TECH (UPC), ESEIAAT, Colom 11, 08222, Terrassa (Barcelona), Spain. \\ pablo.castrillo@upc.edu, joaquim.rigola@upc.edu. URL: www.cttc.upc.edu \\ ${ }^{2}$ Termofluids Av. Jacquard 97-E 08227, Terrassa (Barcelona), Spain. eugenio@ termofluids.com. URL: \\ www.termofluids.com
}

Key words: Fluid-Structure Interaction, Impact Force, Reed Valves, Computing Methods.

\begin{abstract}
The cyclic impact force between a reed valve and the seat plate is the main reason of the valve failure in many thermo-technical devices as compressors, engines, etc. According to experimental observations the latter is due to fatigue and usually occurs in the leading part of the valve 'neck'. In this work, a complex numerical analysis is presented aimed to studying the external forces and internal stresses suffered by the valve. In particular, the impact force between the valve and the seat is studied.

The numerical analysis relies on the coupled synergy of two different simulation concepts. In order to do so, two codes are used: (1) first, the in-house Computational Fluid Dynamics (CFD) code presented in [1] is employed to simulate the Fluid-Structure Interaction (FSI) between gas and valve, extracting reference data for valve displacement and external gas pressures; (2) second, the analysis of the internal structure stresses, together with the impact forces with the plate is implemented in a Computational Solid Dynamics (CSD) code developed in FreeFEM++ [2].

The impact force representation is based on the formulation presented in [3] where a conserving algorithm for frictionless dynamic contact/impact is developed. Due to the importance of obtaining an adequate impact force, an exhaustive study is carried out on its characterization in terms of numerical parameters, such as the penalty stiffness. Under this framework, the valve displacement and impact velocities are verified. Hence, impact forces are analysed in different scenarios, obtaining interesting observations about stresses distribution, with a particular focus on the points where failure is experienced.
\end{abstract}

\section{INTRODUCTION}

When studying the life cycle of a compressor, it is necessary to correctly estimate the impact stresses that are generated due to the impact of the valve against the seat, since it is the latter that generates the failure of the valve $[4,5,6,7]$. Therefore, it has been observed that the failure of the valve occurs in the part that comes into contact with the seat.

In order to perform a numerical analysis of the phenomenon described above, it is necessary to take into account different physics: (1) the fluid, (2) the solid, (3) the interaction between fluid and structure and (4) the simulation of the impact. This not only implies difficulties in mathematical formulation but 
also complex algorithms and high computational costs.

There are different studies where impact stresses are analyzed, in some works the principal stresses $[4,8]$ are analyzed while in others equivalent stresses such as that of Von Mises [9] are taken into consideration. In some cases commercial software are used $[4,8,10]$, while in others in-house codes are developed $[11,9]$. Generally, in these works it is also of interest to obtain the valve displacements as well as the impact velocity [12].

The numerical simulation for the CFD-FSI used in this work was developed on the Termofluids inhouse platform [13] and verified for the framework of reed valves in [1, 14]. The numerical method consists in the combination of a finite volumes solver for the resolution of the flow on an unstructured mesh with a solver for the valve movement. The interface between gas and valve is represented by a moving mesh while the forces are integrated on the valve through a normal mode superposition method with penalty forces to represent the impact between valve and plate.

For the study of principal stresses and Von Mises stresses, an in-house CSD code was developed in the FreeFem ++ tool [2]. With this code it is possible to analyze the stresses, how they change due to the impact between the valve and the seat, as well as the characterization of the impact force. In this simulation only the solid is studied, using the pressure generated by the fluid as a boundary condition. Likewise, in this code the impact force is implemented using the penalty method [3].

The current work is organized as follows. Details on the employed numerical methodologies (CFD and CSD), are given in Section 2. The analysis is performed in dimensionless form (details on physical results can be found in [15]). Results are reported
distribution and the valye stresses variations due to
2 NUMERICAL METHODOLOGIES
2.1 CFID-FSI formulation.
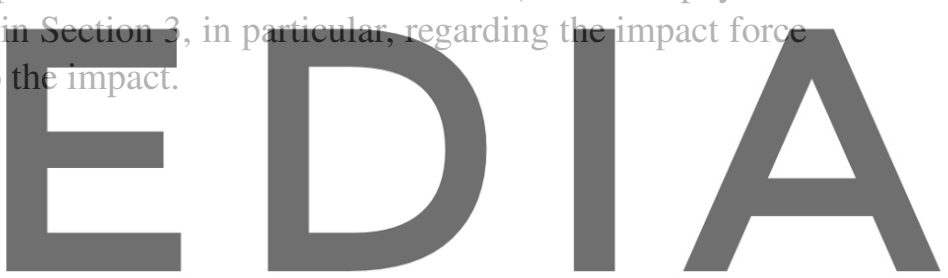

The CFD-FSI method used in this work consists of the complex combination of different simulation

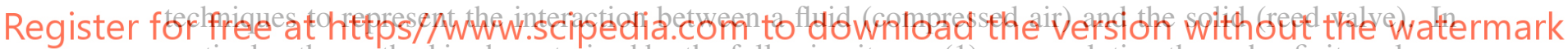
particular, the method is characterized by the following items: (1) gas resolution through a finite volume solver on a moving and unstructured mesh; (2) solving the movement of the solid through a 2D thinplate solver; (3) resolution of the fluid-structure interaction through a semi-implicit approach for strongly coupled problems [16], see Figure 1.

Regarding (1), the Navier-Stokes equations in the incompressibility limit and in a moving mesh framework are solved. A Newtonian behavior of the fluid and constant thermophysical properties are assumed. Hence, the governing equations can be written as follows

$$
\rho \frac{\partial \mathbf{v}}{\partial t}+\rho(\mathbf{c} \cdot \nabla) \mathbf{v}=\mu \nabla^{2} \mathbf{v}-\nabla p,
$$

where $\mathbf{v}$ is the fluid velocity, $p$ the fluid pressure, $\rho$ the fluid density, $\mu$ the fluid viscosity and $t$ the time. $\mathbf{c}$ is the convected air velocity, equal to $\mathbf{v}-\mathbf{v}_{\mathrm{m}}$, with $\mathbf{v}_{\mathrm{m}}$ the velocity of the mesh. A Large-Eddy Simulation (LES) approach is employed: a Sub-Grid Scale (SGS) model is applied to represent the smallest scales, while only the larger eddies are explicitly solved. The chosen SGS scheme is the Wall-Adapting Local Eddy-viscosity (WALE) model. The Navier-Stokes equations are solved by means of a Fractional Step 


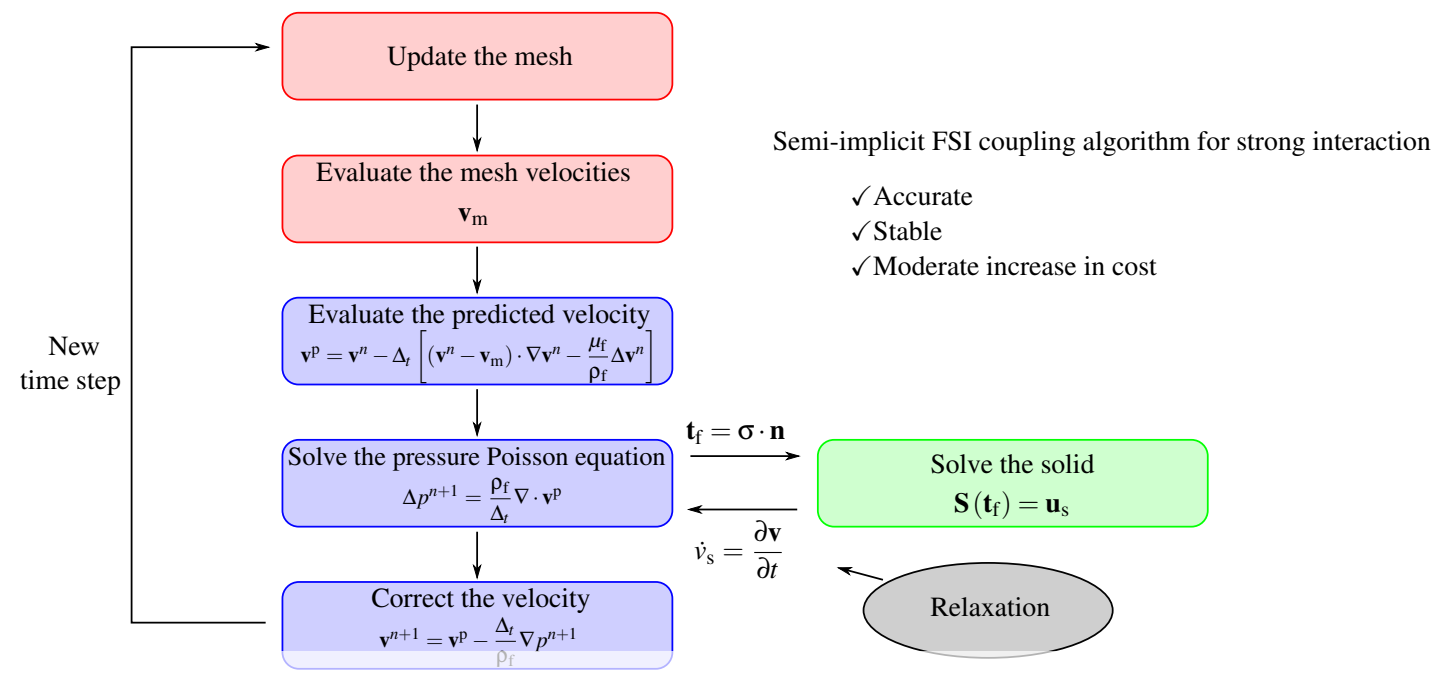

Figure 1: CFD-FSI code flowchart.

Method (FSM) and advanced in time with an explicit scheme, yielding the fluid velocity field at each time step. The domain is represented in a simplified way in Figure 2a, accounting for an inlet port where a variable inlet flow rate is imposed, and lateral outlets with pressure based conditions are set to mimic the flow discharge. The other walls are solid walls boundaries. The base mesh is an unstructured tetrahedral mesh composed by around 300000 cells and represented in Figure 2c. At each time step the mesh undergoes deformations to follow the movement of the valve and to adjust the position of the internal points, thus, accormmodating involving topological alterations. elements of the mesh perto in Figure 2b
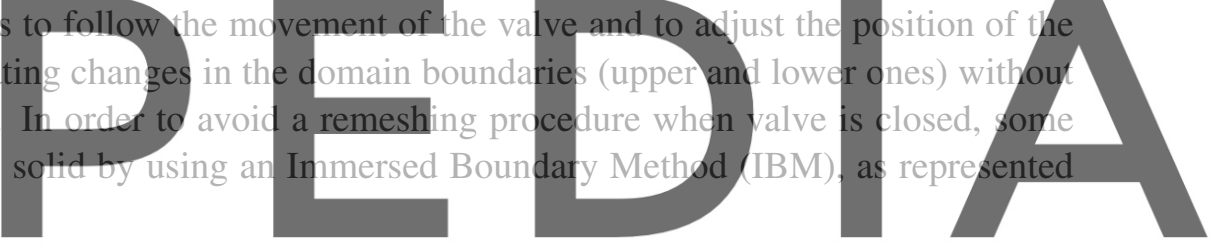
Register for free at https/ Whww.scipedia.com to download the version without the watermark

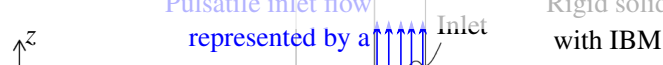

$\stackrel{\uparrow}{\longrightarrow}$ variable flow condition 1 h

with IBM

(a) Domain representation of the CFD-FSI simulation.

(b) Graphic representation of the IBM (grey zone).

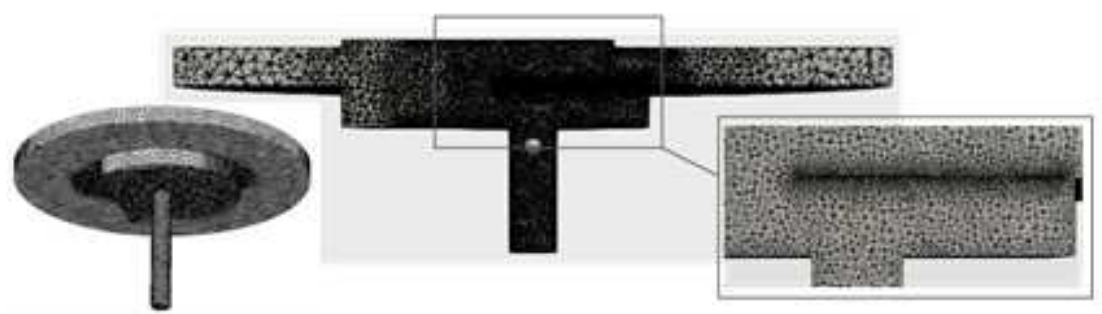

(c) Unstructured tetrahedral mesh composed by around 300000 cells.

Figure 2: CFD-FSI simulation. 
Regarding (2), the motion of the valve (i.e. the boundaries limiting its surface) is modeled by means of the Kirchoff-Love plate theory, valid for arbitrarily shaped plates with uniform thickness $h$. The displacement of this vibrating system can be obtained from a combination of $M$ free vibration modes, as

$$
u_{z}(x, y, t)=\sum_{m=1}^{M} q_{m}(t) \phi_{m}(x, y)
$$

where $\phi_{m}(x, y)$ is the normal deformation pattern and $q_{m}(t)$ is the generalized coordinate corresponding to the vibration mode $m$, respectively. Normal deformation patterns are obtained by means of a commercial software by solving the eigenvalue problem of the free vibration equation of the system, Finally, $q_{m}$ are obtained from the Kirchoff-Love equation by applying the external stresses to the plate, namely, fluid pressure, shear stress and impact force.

Numerical methods corresponding to (1) and (2), together with the coupling algorithm (3) are implemented within the unstructured parallel CFD code TermoFluids [13]. More details about the whole CFD-FSI method are given in [1]. Simulations were run on 64 CPUs of the Joan Francesc Fernandez (JFF) cluster (simulation time around 24 hours), owned by CTTC-UPC.

\subsection{CSD formulation}

In order to study the solid dynamics and its internal stresses the Cauchy moment equations are solved. It is assumed that the material is homogeneous, has a linear elastic behavior and geometric non-linearity effects are not considered. Likgwir governing equations recuces to: where $\rho$ is the valve density, $\mathbf{u}$ is the valve displacement and $\sigma$ is the $\mathrm{C}$ tioned hypotheses the Cauchy tensor is related to the displacement by means of the constitutive equation $\sigma=2 \mu \varepsilon+\lambda \varepsilon$ where $\varepsilon=\frac{1}{2}\left(\nabla \mathbf{u}+\nabla^{\mathrm{T}} \mathbf{u}\right)$ is the infinitesimal strain tensor and $\mu$ and $\lambda$ are the Lamé's maRegister for free at https/fwww scipediacon to downdpad the yersion withqut the watermark and $\left.\mu=\frac{E}{2(i+v)}\right)$.

In order to solve this differential equation, boundary conditions are necessary. In that sense, it is known that the valve is held at one end (Dirichlet conditions), while Neuman conditions are imposed on the others surfaces, in terms of fluid pressures and impact forces. For the fluid pressure $\mathbf{p}$, obtained from the CFD-FSI simulation, the boundary condition is $\sigma \cdot \mathbf{n}=\mathbf{p}$ where $\mathbf{n}$ is the outward normal of the solid. The condition for the impact force is similar to the fluid pressure but it depends on the valve displacement which makes it a non-linear condition. To solve this problem it is necessary to solve the unilateral contact constraints and the persistency condition [3]. The impact between the seat and the valve is considered with the formulation presented in [3]. The normal component of the impact pressure in a time step $\left[t_{n}, t_{n+1}\right]$ is:

$$
I_{\mathrm{p}}=\left\{\begin{array}{lll}
-\frac{U\left(g_{n+1}^{\mathrm{d}}\right)-U\left(g_{n}^{\mathrm{d}}\right)}{g_{n+1}^{\mathrm{d}}-g_{n}^{\mathrm{d}}} & \text { if } & g_{n+1}^{\mathrm{d}} \neq g_{n}^{\mathrm{d}} \\
-U^{\prime}\left(\frac{1}{2}\left(g_{n}^{\mathrm{d}}+g_{n+1}^{\mathrm{d}}\right)\right) & \text { if } \quad g_{n+1}^{\mathrm{d}}=g_{n}^{\mathrm{d}}
\end{array} \text { with } U(g)=\left\{\begin{array}{lll}
\frac{1}{2} k_{\mathrm{p}} g^{2} & \text { if } g \leq 0 \\
0 & \text { if } g>0
\end{array}\right.\right.
$$


where $k_{\mathrm{p}}$ is a penalty parameter and $g^{\mathrm{d}}$ is the dynamic gap. $g^{\mathrm{d}}$ depends on the traditional "real gap" $g$ and in the contact/release logic explained in [3]. Likewise, a parameter $m_{\mathrm{p}}$ is used to reinforce the velocity condition $\dot{g}$ (persistency condition).

For the simulation, a code was developed in the FreeFEM++ tool using a HHT scheme ( $\alpha$-method) for the temporal integration with the parameters $\alpha=0.51, \beta=0.555025$ and $\gamma=0.99$ [3], see Figure 3 .
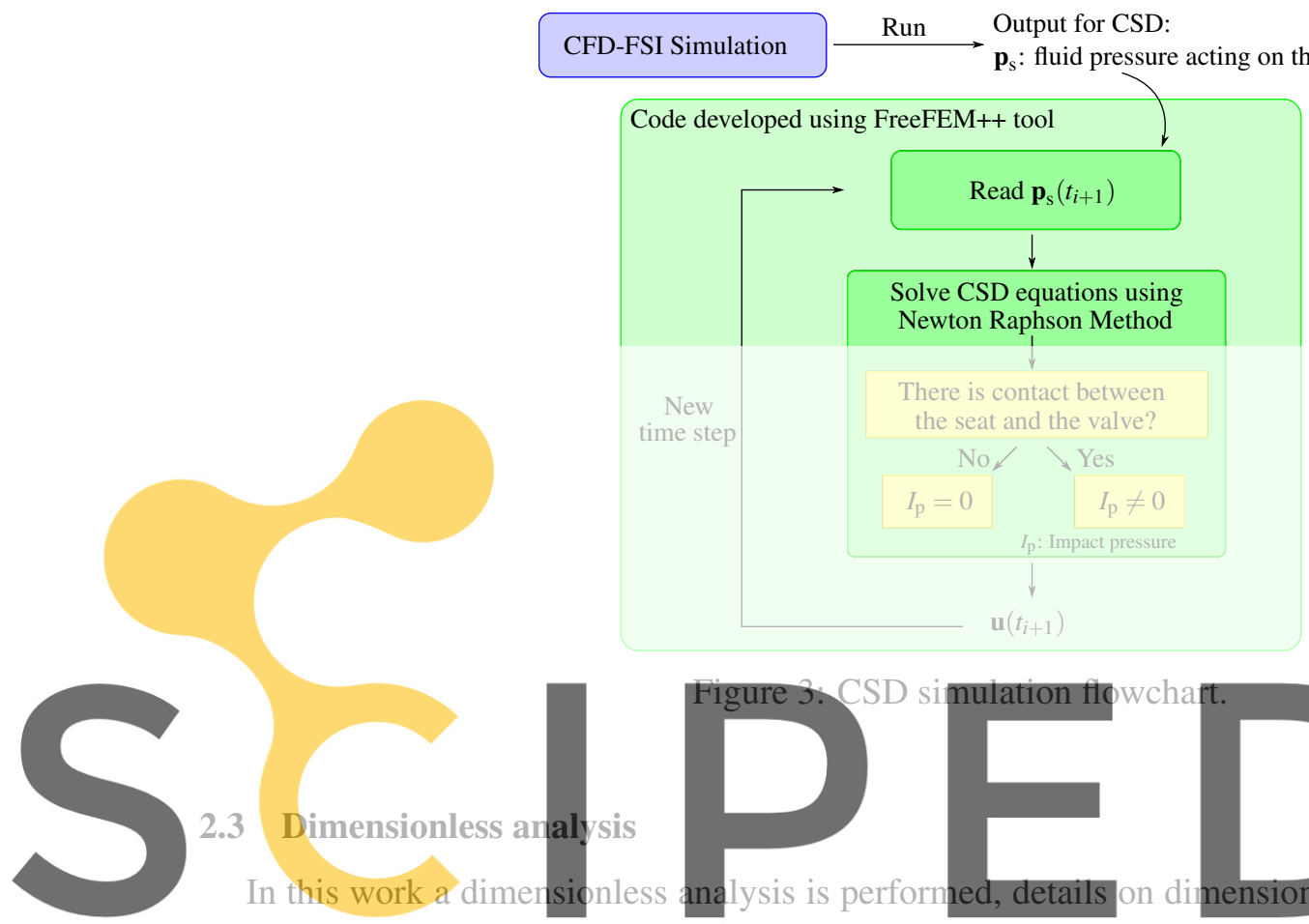

2.3 Dimensionless analysis

In this work a dimensionless analysis is perforned, details
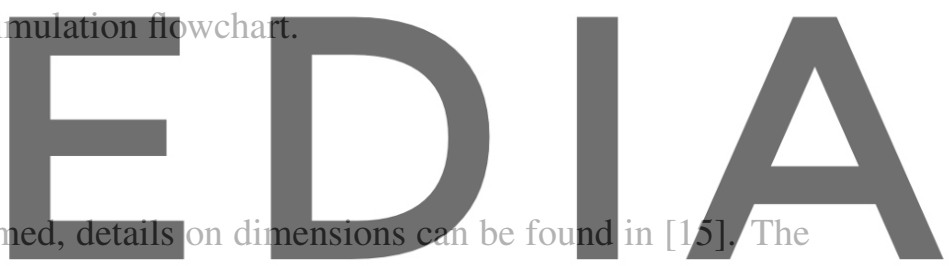

dimensionless magnitudes and mechanical parameters are:

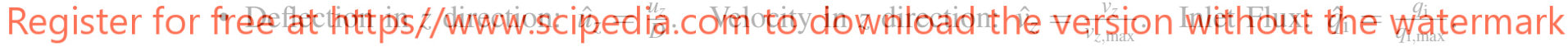

Time: $\hat{t}=\frac{t}{D / v_{z, \max }} . \quad$ Impact parameters: $\hat{k}_{\mathrm{p}}=\frac{k_{\mathrm{p}}}{\rho_{\mathrm{s}} \nu_{z}^{2} \max } / D$ and $\hat{m}_{\mathrm{p}}=\frac{m_{\mathrm{p}}}{\rho_{\mathrm{s}} D} . \quad$ Impact stress $/$ pressure:

$\hat{\sigma}=\frac{\sigma}{\rho_{\mathrm{s}} v_{z, \max }^{2}} . \quad$ Impact Force: $\hat{I}_{\mathrm{F}}=\frac{i_{\mathrm{F}}}{\rho_{\mathrm{s}} v_{z, \max }^{2} D^{2}}$.

- Density of the air: $\hat{\rho}_{\mathrm{f}}=\frac{\rho_{\mathrm{f}}}{\rho_{\mathrm{s}}}=1.10 \times 10^{-3}$. Density of the valve: $\hat{\rho}_{\mathrm{s}}=\frac{\rho_{\mathrm{s}}}{\rho_{\mathrm{s}}}=1$. Viscosity of the air: $\hat{\mu}_{\mathrm{f}}=\frac{\mu}{\rho_{\mathrm{s}} v_{z, \max }^{3} / D}=6.17 \times 10^{-5}$. Young Modulus: $\hat{E}=\frac{E}{\rho_{\mathrm{s}} v_{z, \max }^{2}}=1.39 \times 10^{6} . \quad$ Poisson coefficient: $\hat{v}=\frac{v}{v}=1$.

Where: $D$ is the inlet tube diameter, $q_{\mathrm{i}, \max }$ is the maximum Inlet Flow during the cycle, $v_{z, \max }$ is the maximum velocity of the valve during the cycle and $\rho_{\mathrm{s}}$ is the valve density.

\section{MODEL PARAMETERS SETTING AND RESULTS}

This section presents the results obtained after varying some of the parameters of the problem as well as a discussion of the results in a complete cycle for a certain configuration of the parameters. 


\subsection{Model Parameters Setting}

In this paragraph, displacement and impact velocity are analyzed as a function of the allowed impact area and of the $\hat{k}_{\mathrm{p}}$ parameter. The solution obtained in the CFD-FSI code will be considered to be the reference one since it has been validated with experimental results [15].

\subsubsection{Changing the area of impact}

Although in the experiment the impact area is the entire valve (without the gas inlet orifice) it is decided to analyse what happens when varying the area allowed to impact because in the CFD-FSI model it is observed that the impact force concentrates on the tip of the valve. Therefore, four different possible areas are considered, see Figure 4.

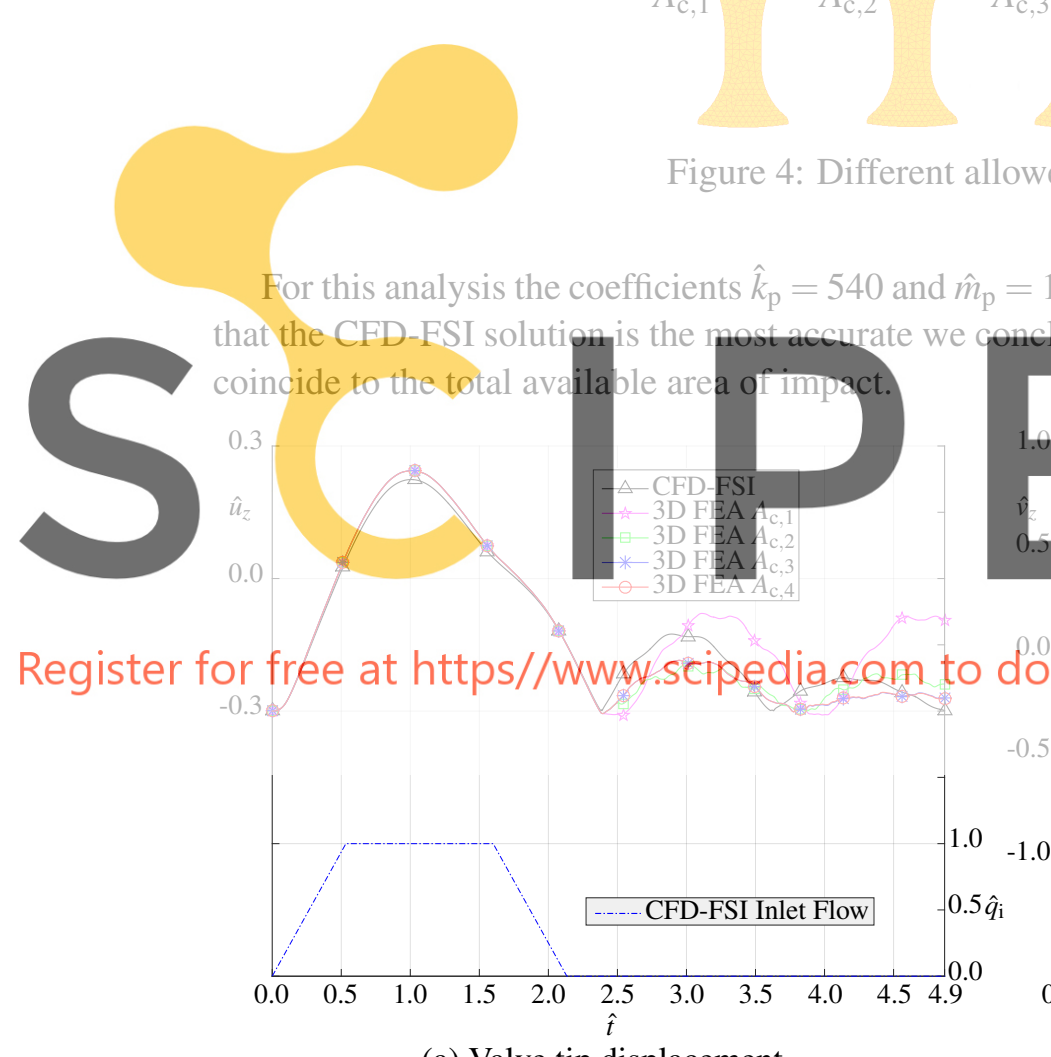

(a) Valve tip displacement.

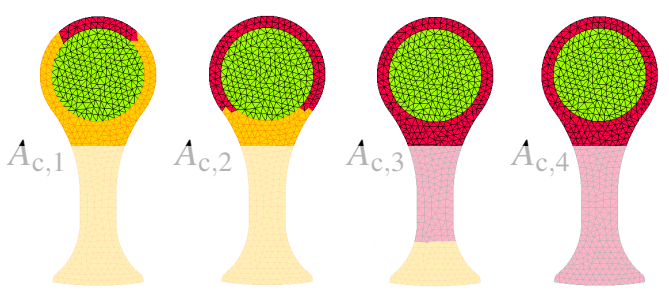

Figure 4: Different allowed areas of impact.

Figure 5: Solution with different allowed areas of impact.

\subsubsection{Varying the impact parameter $k_{p}$}

In this case we analyse how the solution varies by changing the penalty parameter $\hat{k}_{\mathrm{p}}$ using the area selected before $\left(A_{\mathrm{c}, 4}\right)$ and using $\hat{m}_{\mathrm{p}}=1.53 \times 10^{7}$. From Figure 6 it is possible to conclude that when $\hat{k}_{\mathrm{p}}=270$ the best results are obtained. 


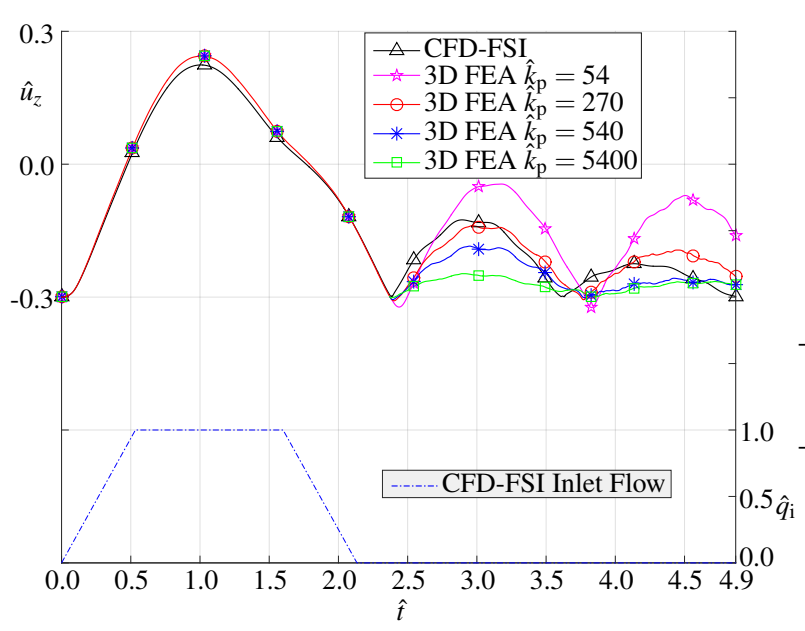

(a) Valve tip displacement.

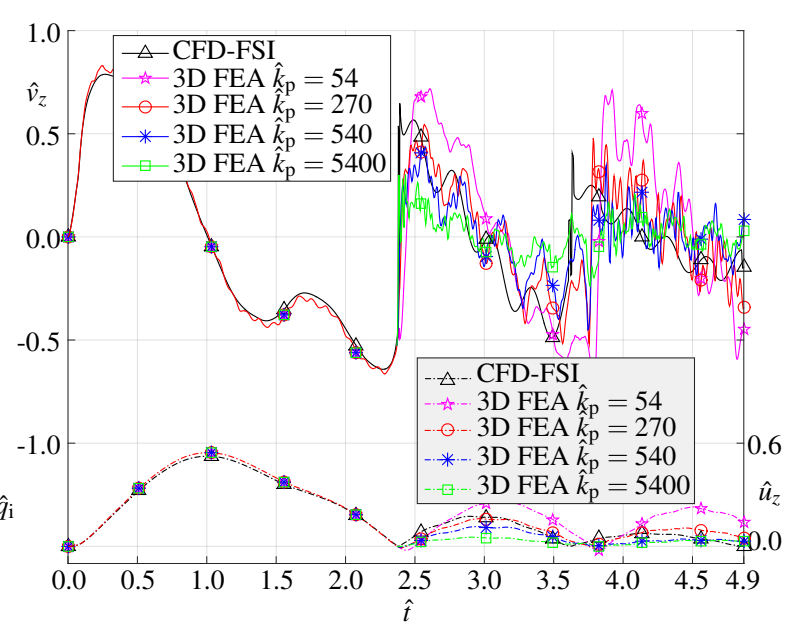

(b) Valve tip velocity.

Figure 6: Solution with different values of penalty parameter $\hat{k}_{\mathrm{p}}$.

\subsection{Physical results}

As a consequence of the preliminary parameter analysis, it is decided to use $A_{c, 4}$ as contact area and $\hat{k}_{\mathrm{p}}=270$. Hence, the results in terms of displacement and velocity are reported in the plots of Figure 7 .
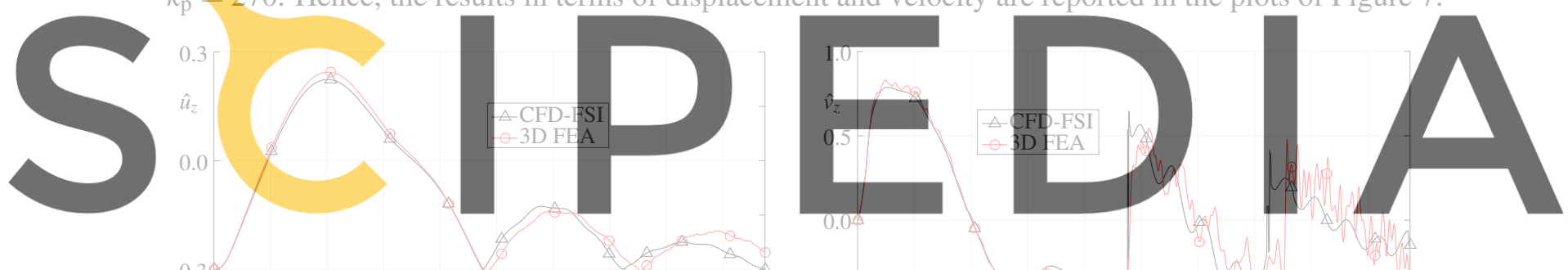

Register for free at https//www.scipedia.com to download the version without the watermark

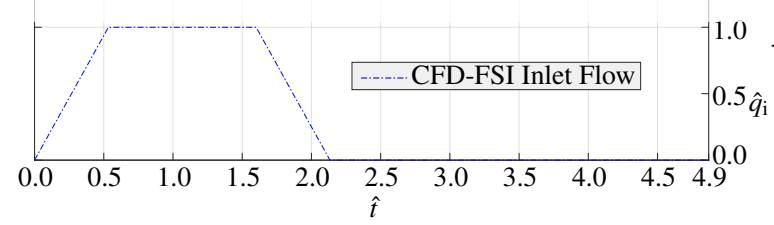

(a) Valve tip displacement.

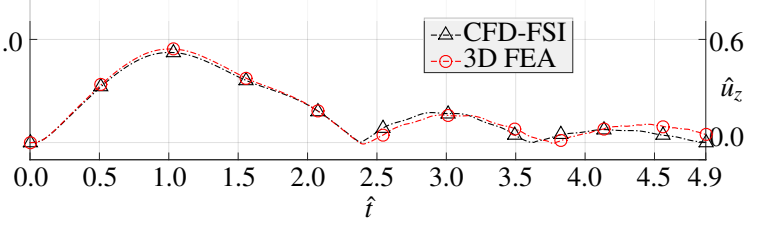

(b) Valve tip velocity.

Figure 7: Solution for the selected area of impact and the selected impact parameter.

\subsubsection{Results for the first impact}

The first impact occurs in the time interval $\hat{t}=(2.38,2.44)$ which corresponds to the interval $460-$ 480 of the simulation. In Figure 8a it is possible to see the impact pressure distribution for all interval instants. The following conclusions can be drawn: (1) The first impact begins at the tip of the valve. (2) 
Then the impact pressure is distributed over the valve surface. (3) Finally the valve is released from the seat. Likewise, in Figure $8 \mathrm{~b}$ the impact force and the maximum impact pressure are represented.

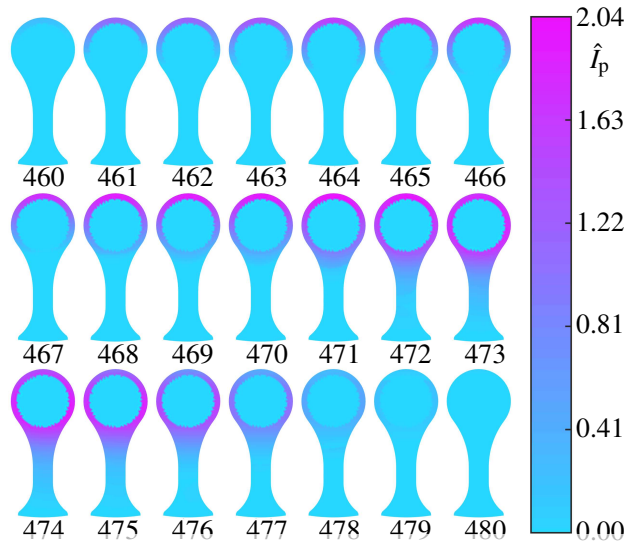

(a) Impact pressure distribution.

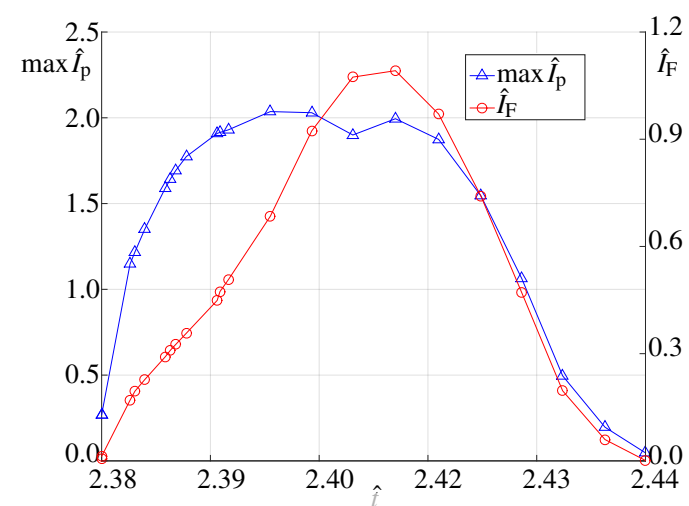

(b) Impact force.

Figure 8: Impact pressure distribution and impact force for all interval instants in the first impact.

In Figure 9 the principal stresses $\hat{\sigma}_{I} \geq \hat{\sigma}_{I I I}$ and the equivalent Von Mises stress $\hat{\sigma}_{\mathrm{vm}}$ for the first impact can be observed. It is clear that before impact, due to bending, the highest stresses are found at the valve neck. Nevertheless, during the impact, the highest stresses are mainly at the valve tip. Also, it is interesting to observe how lower stresses, which are initially close to 0 due to bending, suddenly increase

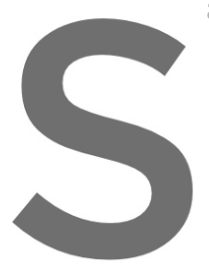
as a consequence of the impact.
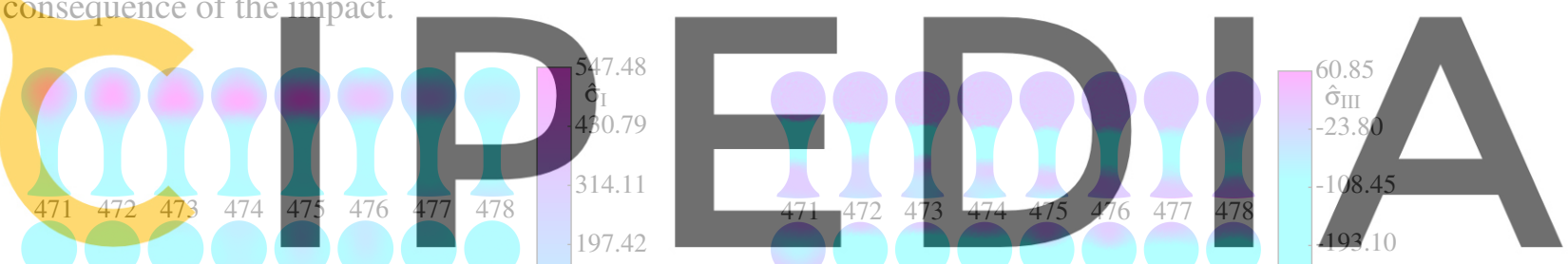

Register for free at https//www.scipedia.com to download the version without the watermark

(a) Principal stress $\hat{\sigma}_{I}$. (b) Principal stress $\hat{\sigma}_{\text {III. }}$

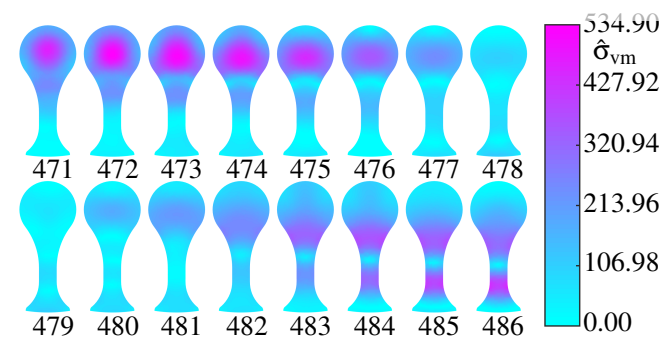

(c) Von Mises equivalent stress $\hat{\sigma}_{\mathrm{vm}}$.

Figure 9: Principal stresses $\left(\hat{\sigma}_{I}, \hat{\sigma}_{I I I}\right)$ and Von Mises equivalent stress $\hat{\sigma}_{\mathrm{vm}}$ for the first impact.

\subsubsection{Results for the second impact}

The second impact occurs in the time interval $\hat{t}=(3.69,3.80)$ which corresponds to the interval $768-790$ of the simulation. In Figure 10a it is possible to see the impact pressure distribution for all 
interval instants. From it, it is possible to conclude that: (1) The second impact begins at the clamping of the valve spreading towards the tip. (2) Then the pressure drops and the impact is again at the tip but on a smaller surface. (3) Finally the valve is released from the seat. On the other hand, in Figure 10b the impact force and the maximum impact pressure are represented.

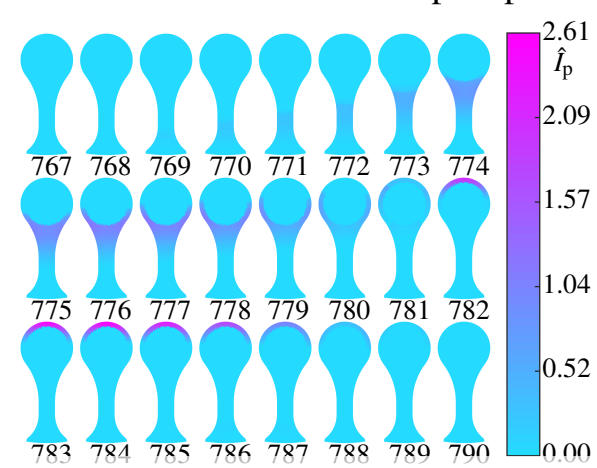

(a) Impact pressure distribution.

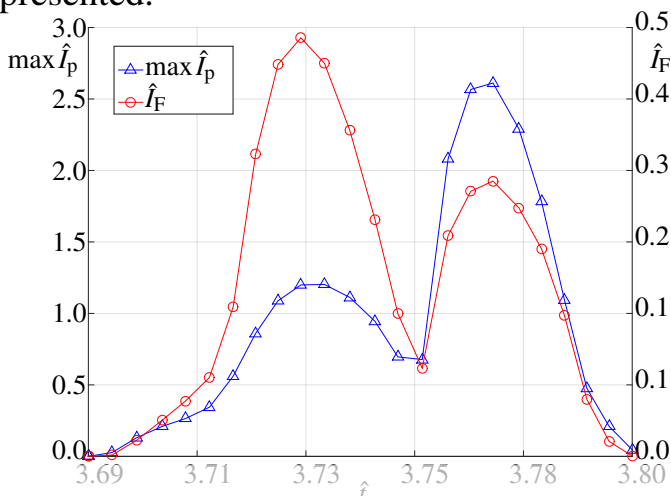

(b) Impact force.

Figure 10: Impact pressure distribution and impact force for all interval instants in the second impact.

In Figure 11 the principal stresses $\hat{\sigma}_{\mathrm{I}} \geq \hat{\sigma}_{\mathrm{III}}$ and the equivalent Von Mises stress $\hat{\sigma}_{\mathrm{Vm}}$ for the second impact can be observed. In this case the conclusions are similar to those of the first impact as you may see in the different figures. It is worth mentioned that an increase compared to the first impact is noted in the three studied stresses. This is explained because, despite of having lower impact forces, the impact
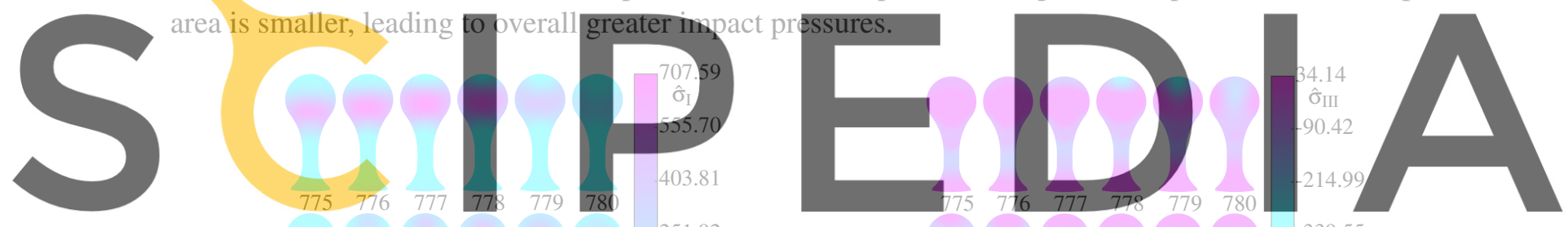

Register for free at https//www.scipedia.com to download the version withoutthe watermark

(a) Principal stress $\hat{\sigma}_{1}$.

(b) Principal stress $\hat{\sigma}_{\text {ili }}$.

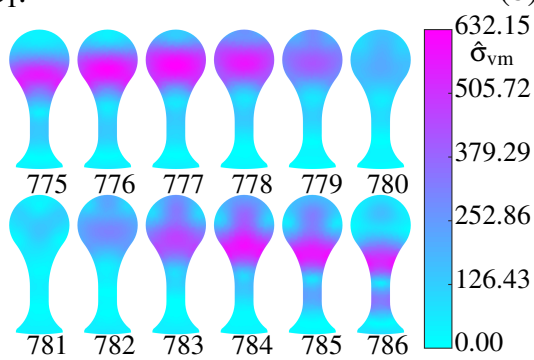

(c) Von Mises equivalent stress $\hat{\sigma}_{\mathrm{vm}}$

Figure 11: Principal stresses $\left(\hat{\sigma}_{\mathrm{I}}, \hat{\sigma}_{\mathrm{III}}\right)$ and Von Mises equivalent stress $\hat{\sigma}_{\mathrm{vm}}$ for the second impact. 


\subsection{Envolvent of Von Mises and principal stresses in some points.}

In Figure 12 it is possible to observe how stresses change throughout the cycle in four points where the valve usually fails, as it has been mentioned before $[4,5,6,7]$. It is interesting to mention after analyzing the graphics that in all cases the stresses increase due to impact, particulary the lower stresses $\left(\sigma_{\text {III }}\right)$, shown in Figure 12d.

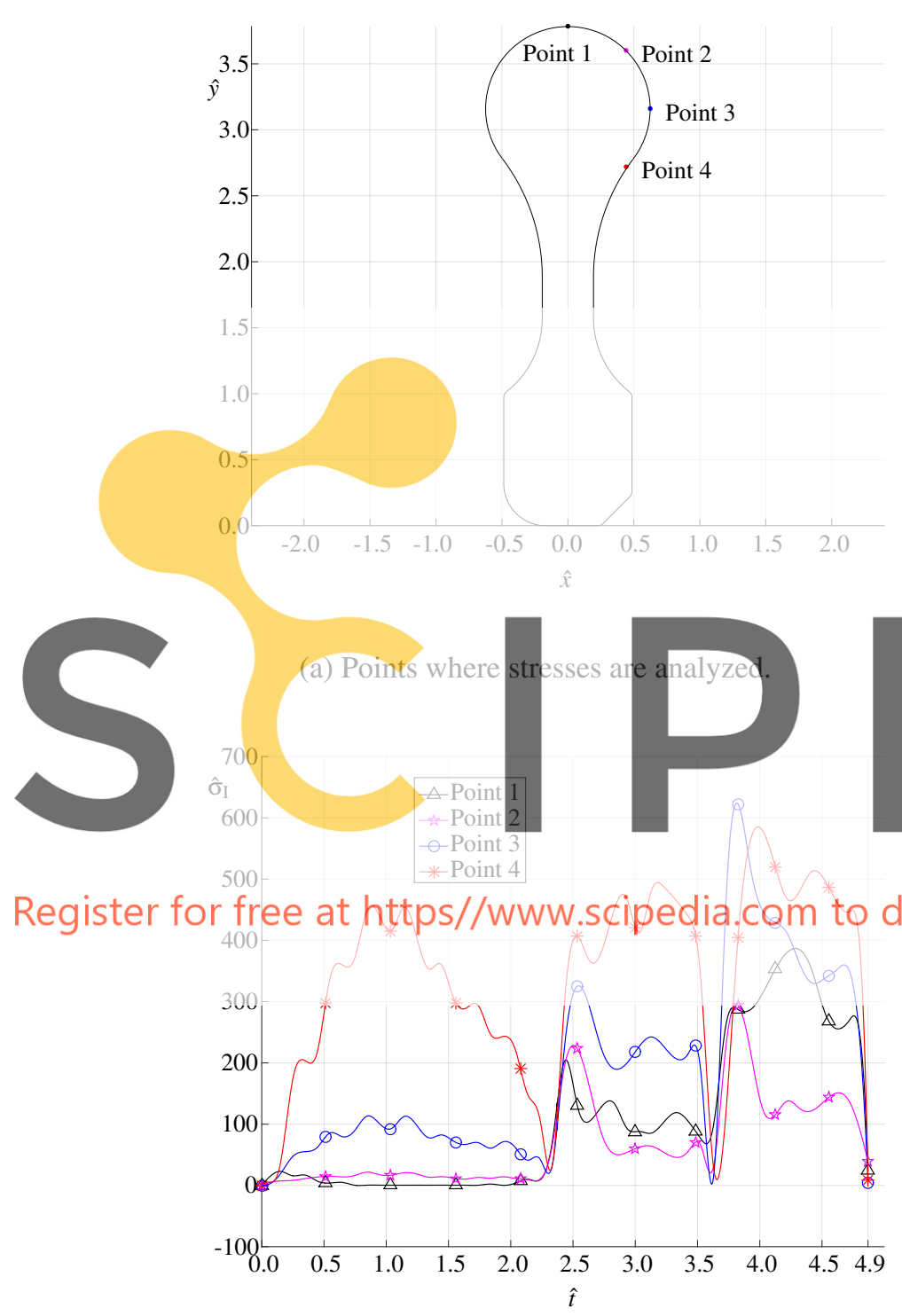

(c) Principal stress $\hat{\sigma}_{\mathrm{I}}$.
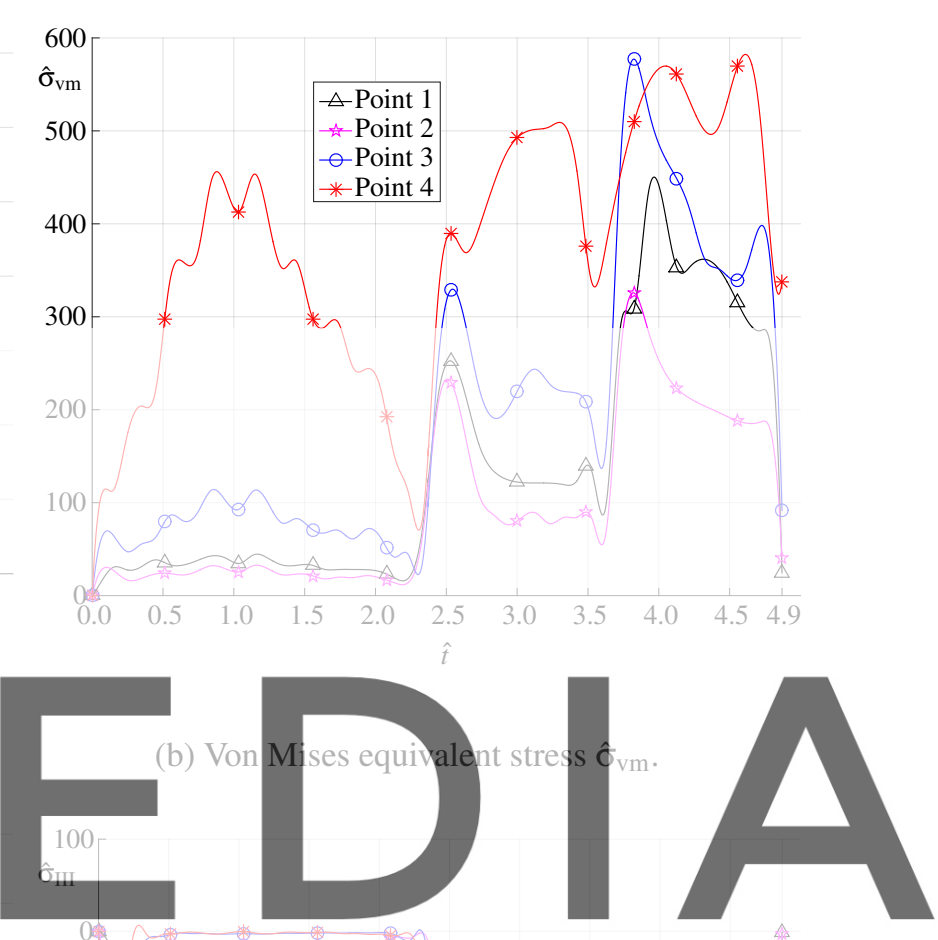

free at https//www.scipedia.com to domnload the version without the watermark

Figure 12: Envolvent of Von Mises and principal stresses in some points. 


\section{ACKNOWLEDGMENT}

The authors acknowledge Voestalpine Precision Strip AB company for the previous research collaboration project that allowed to validate experimentally the presented numerical methods. P. Castrillo gratefully acknowledges the Universitat Politècnica de Catalunya and Banco Santander for the financial support of his predoctoral grant FPI-UPC (109 FPI-UPC 2018). E. Schillaci acknowledges the financial support of the Programa Torres Quevedo (PTQ2018-010060). This work has also been financially supported by a competitive R+D project (ENE2017-88697-R) by the Spanish Research Agency.

\section{CONCLUSIONS}

In this work a coupled CFD-CSD methodology is employed to analyze the fluid-structure interaction between a compressed gas flow and a metal valve. We proposed an implementation to evaluate the impact pressures that are generated between valve and seat on the CSD model, based on a dynamic contact/impact conservative algorithm using a penalty method. In Section 3.1 we analysed the effect of model parameters such as the penalty coefficient or the allowed area of impact. In the physical analysis, presented in Section 3.2, we performed an analysis of stresses variations due to the effect of the valve hitting the seat. As a consequence, it is possible to conclude that at the points where the valve usually fails, stresses increase dramatically due to impact, being 100-200 times higher than bending stresses. Finally, it is observed that $\hat{\sigma}_{\text {III }}$ (lower principal stress) at the bottom surface increases in absolute value significantly due to impact. The coupled methodology demonstrated to be a reliable tool to analyse the stresses generated on the valve along its complete working cycle, therefore proving to be an adequate tool for analysis of additional valves, e.g. in terms of different shapes and materials.

\section{REFERENCES}

[1] I. Gonzalez, A. Naseri, J. Rigola, C. D. Pérez-Segarra, and A. Oliva, "Detailed prediction of fluidsolid coupled phenomena of turbulent flow through reed valves," in In IOP Conference Series: Materials Science and Engineering, vol. 604, p. 012064, 2019.

[2] F. Hecht, "New development in freefem+," Journal of Numerical Mathematics, vol. 20, no. 3-4, pp. 251-265, 2012.

[3] F. Armero and E. Petocz, "Formulation and analysis of conserving algorithms for frictionless dynamic contact/impact problems," Computer Methods in Applied Mechanics and Engineering, vol. 158 , no. 3-4, pp. 269-300, 1998.

[4] X. Yu, Q. Tan, Y. Ren, X. Jia, and L. Jin, "Numerical Study of the Reed Valve Impact in the Rotary Compressor by FSI Model,” Energy Procedia, vol. 105, pp. 4890-4897, 2017.

[5] W. M. Tofique, A. Löf, C. Millward, and A. Nawaz, "Investigation of the influence of valve reedimpact seat plate interaction on their impact fatigue properties," International Compressor Engineering Conference, Purdue University, no. 1976, pp. 1-11, 2018.

[6] S.-W. Woo and D. L. O'Neal, "Improving the Reliability of a Domestic Refrigerator Compressor Subjected to Repetitive Loading," Engineering, vol. 08, no. 03, pp. 99-115, 2016.

[7] M. Hareland, A. Hoel, S. Jonsson, and D. Liang, "Selection of Flapper Valve Steel for High Efficient Compressor," International Compressor Engineering Conference, pp. 1-9, 2014. 
[8] F. C. Lajús, C. J. Deschamps, and M. Alves, Numerical analysis of seat impact of reed type valves. No. 1, Woodhead Publishing Limited, 2013.

[9] Jo. Nilsson, L. Nilsson, and M. Oldenburg, "Impact stresses in flapper valves - a finite element analysis.," International Compressor Engineering Conference, 1980.

[10] X. Yu, Y. Ren, Q. Tan, Z. Lu, X. Jia, and X. Wang, "Study on the torsional movement of a reed valve in a rotary compressor," Advances in Mechanical Engineering, vol. 10, no. 6, pp. 1-10, 2018.

[11] Y. Lee and S. Son, "Study on the Fatigue Strength of a Suction Flapper Valve used in a High Efficient Reciprocating Compressor 2 . DESIGN PARAMETERS OF SUCTION VALVEG," International Compressor Engineering Conference, pp. 1-6, 2008.

[12] Y. Wang, C. Xue, J. Feng, and X. Peng, "Experimental investigation on valve impact velocity and inclining motion of a reciprocating compressor," Applied Thermal Engineering, vol. 61, no. 2, pp. 149-156, 2013.

[13] “Termo fluids s.l., www.termofluids.com,” 2020.

[14] I. Gonzalez, O. Lehmkuhl, A. Naseri, J. Rigola, and O. Assensi, "Fluid-Structure Interaction of a Reed Type Valve Subjected to Piston Displacement," International Compressor Engineering Conference, pp. 1-10, 2016.

[15] M. W. Tofique, A. Löf, E. Schillaci, P. Castrillo, and R. Joaquim, "Experimental and numerical analysis of reed valve movement in an impact fatigue test system and reciprocating compressors," International Compressor Engineering Conference at Purdue, Submitted/Under review., 2021.

[16] A. Naseri, O. Lehmkuhl, I. Gonzalez, E. Bartrons, C. D. Pérez-Segarra, and A. Oliva, "A semiimplicit coupling technique for fluid-structure interaction problems with strong added-mass effect," Journal of Fluids and Structures, vol. 80, pp. 94-112, 2018. 